\title{
Role of MRI in planning radical prostatectomy. What is the added value?
}

${\text { Jose Marenco(1), Clement } \operatorname{Orczyk}^{(1,2)} \text {, Tom Collins(1), Caroline Moore }}^{(1,2)}$, Mark Emberton ${ }^{(1,2)}$

\section{Affiliations}

1. University College London Hospital, NHS Foundation Trust, London, UK.

2. University College of London. Division of surgery and interventional science

\section{Corresponding author}

Jose Luis Marenco Jimenez

$+(44) 7539201032$

jlmarencoj@gmail.com

47 wimpole street, Marylebone, London. UK

W1G 8SE

\section{Funding sources}

The authors confirm that all the funded sources were the following: None. 


\section{Role of MRI in planning radical prostatectomy. What is the added value?}

\section{Introduction}

The goal of radical prostatectomy is eradicating oncological disease while achieving the best possible functional outcomes. In this sense, nerve-sparing offer a greater chance of potency recovery after surgery. Accurately locating prostate cancer foci is instrumental to identify good candidates for this approach whilst keeping a safe rate of oncological margins.

Furthermore, prediction of extra-capsular extension or seminal vesicles involvement during pre-surgical planning can help to adjust excisional margins.

\section{Method}

A literature search of the MEDLINE/PubMed and Scopus database was performed. A peer review of the Journals took place to select relevant articles

\section{Results}

Mp-MRI has proof to be accurate to detect and locate prostate cancer. Sensibility to detect extra-capsular extension, seminal vesicle involvement or T3 in general is moderate, alas with a great specificity when detected. Measurement of membranous urethral length has shown to be useful in predicting probability of achieving continence after surgery. Furthermore, image guided surgery has been tested to be accurate to direct surgical planes in order to safely preserve neurovascular bundles

\section{Conclusion}

The use of Mp-MRI for pre-surgical planning allows tailoring surgery in order to achieve optimal functional outcomes whilst not compromising positive surgical margins rate. 


\section{Introduction}

Radical prostatectomy (RP) is considered the standard surgical approach to localised prostate cancer. Traditionally pre-operative prostate cancer assessment was performed based on nomograms ${ }^{1}$. MRI has shown to increase accuracy of clinical nomograms to predict final pathological staging.

The goal of RP is achieve cure, whilst keeping the lowest possible rate of side effects. In this sense there is a balance between wide excision, which will theoretically achieve the lowest rate of positive surgical margins, and a narrower excision trying to preserve structures that will implement functional structures. For this strategy, a trustable way to determine the likelihood of disease in the boundaries of the prostate is mandatory. To address this dilemma mp-MRI has shown to be effective in detecting and locating prostate cancer to a great degree of certainty ${ }^{10 .}$

Another essential goal in radical prostatectomy is maintaining urinary continence. Greater Maximal urethral length (MUL) prior to RP is associated with higher degrees of continence. Thus, preoperative measurement is recommended.

\section{Staging of localised prostate cancer}

Local staging of prostate cancer is paramount to offer an adequate treatment. Previously, this was done on the grounds on nomograms integrating PSA, Gleason score, number of positive scores and clinical stage based on digital rectal examination. This information is blind to size and location of tumour as well as relation with surrounding organs. Moreover, digital rectal examination is subjective and operator dependant ${ }^{8}$ and often underestimates tumour extension. The value of Mp-MRI in local staging is unclear. In a recent systematic review, high specificity but inconstant and generally low sensitivity was found for Mp-MRI to detect extra-capsular extension, seminal vesicle invasion and $\mathrm{T}_{3}$ stage $^{11}$. Moreover, Mp-MRI allows to detect bulky extra-capsular extension, albeit not micro-capsular breach, and therefore is a more reliable method for local staging prior to RP4(ref-MRI more reliable staging).

A reliable prediction of final pathological stage is of seminal importance given the recent trend towards selecting more high risk prostate cancer for radical prostatectomy ${ }^{9}$, where involvement of bladder neck, peri-prostatic fat or surrounding organs is not rare.

\section{Pre-surgical nodal evaluation}

The most reliable way to assess lymph node involvement is a pelvic lymph node dissection (PLND); this is of course not free of morbidity or invasiveness.

Therefore, identification of patients that will benefit from pelvic lymph node dissection and the extent of such is another preoperative planning where imaging might change the current decision-making tools.

On a recent meta-analysis, the pooled sensitivity within different studies for both CT scan and MRI scan was low at 0.42 and 0.39 respectively. Specificity was 0.82 for both tests. There was no statistical difference between the two tests, hence both have a similar poor capacity to detect nodal involvement. This is basically because both scans evaluate only anatomical information, lacking metabolic data. 
Adding clinical parameters to the MRI findings seem to increase the ability to predict LNI (AUC 0.956). Furthermore, tumour volume, tumour ADC value and tumour T stage were the most accurate independent predictors of LNI. Finally, the prevalence of nodal metastasis in patients with localised (T2 on MRI) tumours with tumour size $<1 \mathrm{~cm}$ was very low; suggesting that MRI could be used to triage which patients might avoid PLND.

Nevertheless, consi

\section{Role of MRI to guide nerve sparing radical prostatectomy}

Preservation of neurovascular bundles (NVB) has shown to improve functional outcomes after radical prostatectomy. Despite anatomical variations that make the degree of preservation hard to predict(ref. anatomical variations,5), in a general fashion NVB are considered to run posterolateral to the prostate from the base to the apex. As discussed above, the gain in side effects reduction must be weighted against the risk of compromising surgical margins, thus increasing the risk of prostate cancer recurrence and the morbidity of adjuvant treatments. Therefore in order to plan a narrower excision plane, it is desirable to ensure the absence of disease close to the posterolateral margins of the prostate. The role of Mp-MRI to decide the level of nerve spare and select patients for this approached was evaluated by Riccardo et al(Ref). They performed an MRI scan on 137 men deemed to undergo radical prostatectomy. The previous nerve sparing strategy based on clinical data was revised with the MRI findings, approximately in half the cases the nerve sparing strategy was changed either at patient or side level. Appropriateness of resection was considered taking into account presence of extra-capsular extension or positive surgical margins on the side were the NS strategy was changed. Overall appropriateness of change was referred to be $70 \%$. Moreover, they demonstrated lower positive margin rates on this cohort of patients when compared to a synchronous dataset of RARP ${ }^{7}$.

Further, Panebianco et al. selected 125 patients who were deemed to have bilateral nerve sparing radical prostatectomy (NS RP) based on clinical parameters and also performed an MRI scan. Patients were subsequently divided according to MRI findings into those eligible for bilateral NS or those where the strategy should change to either unilateral or no NS bilateral, in roughly $30 \%$ of men the initial bilateral NS RP plan was changed. The authors claim that the use of pre-operative Mp-MRI allowed choosing an appropriate level of excision in $95.9 \%$ and $87.5 \%$ of men in the bilateral and the uni or non NS RP respectively. Moreover, the rate of positive margins in the posterolateral region was low at $3.8 \%{ }^{3}$.

\section{Measurement of membranous urethral length}

Despite surgical advancements, incidence of urinary incontinence after radical prostatectomy is high, particularly in the early postoperative period. Recovery of continence, is unpredictable and quite variable amongst different series(REF). Time to continence recovery is also variable with improvements reported up to two years after surgery.

Various patient-related risk factors that affect continence have been reported. Amongst them, membranous urethra length (MUL) has been hypothesized to be positively correlated with better recovery of urinary continence. In one recent meta-analysis, greater MUL, measured on T2 weighted images on preoperative 
MRI, correlated with better continence results at 3, 6 and 12 months. Furthermore, after statistical analysis every extra centimetre of MUL improved likelihood of continence recovery by more than $200 \%{ }^{6}$.

Therefore, preoperative measurement of MUL should be a standard preoperative evaluation which will add valuable information for patient counselling.

\section{Conclusion}

Radical prostatectomy is the mainstay treatment for localised prostate cancer.

Despite technological improvements, functional outcomes after radical prostatectomy are still variable and in some cases unpredictable. The introduction of preoperative imaging can shift the trend towards a more tailored radical prostatectomy incorporating patient and tumour information on the pre-surgical planning. This can reduce functional impact of the procedure without compromising oncological safety for a better-counselled patient. 


\section{References}

1. Memorial sloan kettering cancer center. Prostate cancer nomograms preradical prostatectomy. http://www.mskcc .org/nomograms/prost ate/pre_op. Accessed Jul 2018

2. Yuh B, Artibani W, Heidenreich A, Kimm S, Menon M, Novara G et al. The Role of Robot-assisted Radical Prostatectomy and Pelvic Lymph Node Dissection in the Management of High-risk Prostate Cancer: A Systematic Review. European Urology. 2014;65(5):918-927

3. PANEBIANCO, $\mathrm{V}$. et al. Use of multiparametric MR with neurovascular bundle evaluation to optimize the oncological and functional management of patients considered for nerve-sparing radical prostatectomy. J Sex Med, v. 9, n. 8, p. 2157-66, Aug 2012. ISSN 1743-6109. Disponível em: < https://www.ncbi.nlm.nih.gov/pubmed/22642466 >.

4. Wang L, Hricak H, Kattan M, Chen H, Scardino P, Kuroiwa K. Prediction of Organ-confined Prostate Cancer: Incremental Value of MR Imaging and MR Spectroscopic Imaging to Staging Nomograms. Radiology. 2006;238(2):597603.

5. Kiyoshima K, Yokomizo A, Yoshida T, Tomita K, Yonemasu H, Nakamura M, Oda Y, Naito S, Hasegawa Y. Anatomical features of periprostatic tissue and its surroundings: A histological analysis of 79 radical retropubic prostatectomy specimens. Jpn J Clin Oncol 2004;34:463-8.

6. Mungovan S, Sandhu J, Akin O, Smart N, Graham P, Patel M. Preoperative Membranous Urethral Length Measurement and Continence Recovery Following Radical Prostatectomy: A Systematic Review and Meta-analysis. European Urology. 2017;71(3):368-378.

7. Schiavina R, Bianchi L, Borghesi M, Dababneh H, Chessa F, Pultrone C et al. MRI Displays the Prostatic Cancer Anatomy and Improves the Bundles Management Before Robot-Assisted Radical Prostatectomy. Journal of Endourology. 2018;32(4):315-321.

8. Spigelman S, McNeal J, Freiha F, Stamey T. Rectal Examination in Volume Determination of Carcinoma of the Prostate: Clinical and Anatomical Correlations. The Journal of Urology. 1986;136(6):1228-1230.

9. Weiner A, Matulewicz R, Schaeffer E, Liauw S, Feinglass J, Eggener S. Contemporary management of men with high-risk localized prostate cancer in the United States. Prostate Cancer and Prostatic Diseases. 2017;20(4):442-442.

10. Ahmed H, El-Shater Bosaily A, Brown L, Gabe R, Kaplan R, Parmar M et al. Diagnostic accuracy of multi-parametric MRI and TRUS biopsy in prostate cancer (PROMIS): a paired validating confirmatory study. The Lancet. 2017;389(10071):815-822.

11. Accuracy of Magnetic Resonance Imaging for Local Staging of Prostate Cancer: A Diagnostic Meta-analysis Maarten de Rooij *, Esther H.J. Hamoen, J. Alfred Witjes, Jelle O. Barentsz, Maroeska

\section{to include}

1. Nerve-sparing approach during radical prostatectomy is strongly associated with the rate of postoperative urinary continence recovery 
2. Preoperative Membranous Urethral Length Measurement and Continence Recovery Following Radical Prostatectomy: A Systematic Review and Meta-analysis

3. Spigelman, S.S., et al. Rectal examination in volume determination of carcinoma of the prostate: clinical and anatomical correlations. J Urol, 1986. 136: 1228. https://www.ncbi.nlm.nih.gov/pubmed/3773095

4. Feng TS, Sharif-Afshar AR, Wu J et al (2015) Multiparametric MRI improves accuracy of clinical nomograms for predicting ext- racapsular extension of prostate cancer. Urology. 86:332-337

5. Coakley FV, Eberhardt S, KattanMW,Wei DC, Scardino PT, Hricak H. Urinary continence after radical retropubic prostatectomy: relationship relationship with membranous urethral length on preoperative endorectal magnetic resonance imaging. JUrol 2002;168:1032-5.

6. Paparel P, Akin O, Sandhu JS, et al. Recovery of urinary continence after radical prostatectomy: association with urethral length and urethral fibrosis measured by preoperative and postoperative endorectal magnetic resonance imaging. Eur Urol 2009;55: 629-37. 